\title{
Posture paradoxale: Bilan de recherche sur le travail des médiateurs et médiatrices de l'art contemporain dans le milieu muséal
}

\author{
Marilyn Farley, Concordia University \\ marilynfarley@outlook.com
}

Résumé Cet article résume les résultats d'une recherche exploratoire menée en 2014 et 2015 , portant sur les expériences de terrain des médiatrices et médiateurs de l'art contemporain dans le contexte muséal. Les témoignages de six participant(e)s mettent en lumière les rôles et les réalités des médiateur(trice)s, mais aussi les défis et les tensions auxquels leur profession fait actuellement face. Le projet de recherche Réconcilier l'irréconciliable : L'expérience de terrain des médiatrices et médiateurs de l'art contemporain (2015) ainsi que le présent article visent à faire connaître les perspectives uniques des médiateur(trice)s et à participer à la valorisation de leurs voix comme acteurs culturels importants.

Mots clés Médiation; art contemporain; expériences des médiatrices et médiateurs; définitions et défis.

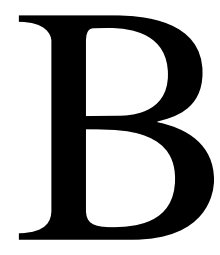

ien que les écrits sur la médiation de l'art contemporain se soient multipliés au cours des dernières années, peu se concentrent sur les expériences vécues des médiatrices et médiateurs oeuvrant dans le contexte muséal. Le projet de recherche Réconcilier l'irréconciliable (Farley, 2015) visait (humblement) à remédier à cette situation en mettant en lumière les rôles et actions des médiateur(trice)s à partir de leurs observations et de leurs expériences du «terrain». Cette recherche exploratoire permit de revisiter plusieurs définitions déjà admises de la médiation en contexte muséal, mais également de nuancer et d'ajouter certains aspects. Dans le cadre de cet article, je traiterai de quelques-uns des éléments de définition amenés par les sujets-participants. En me basant sur leurs propos, je discuterai des objectifs de ces médiateur(trices) de l'art contemporain et des défis/tensions qu'ils rencontrent dans le cadre de leurs fonctions.

\section{Méthodologie et procédures}

Afin d'ancrer la recherche dans l'expérience personnelle des sujets-participants, j'ai opté 
pour une méthode d'analyse en aval, en procédant à partir des données brutes («bottom-up coding ») (Urquhart, 2013). L'analyse fut réalisée en suivant les étapes de la théorie ancrée ( " grounded theory»), permettant ainsi l'émergence de concepts à partir des données ellesmêmes (Stern et Porr, 2011). Par la suite, ces concepts furent mis en relation avec les recherches antérieures et la littérature existante. La collecte de données fut effectuée auprès de six médiateur(trices), divisé(e)s en deux groupes de discussion de trois personnes, lors desquels j'occupai le rôle d'animatrice. Lors de ces rencontres - enregistrées, puis entièrement retranscrites - les sujets-participants furent invités à partager leurs expériences de terrain et leurs observations sur la médiation. Quelques questions choisies au préalable permirent de démarrer la discussion, mais rapidement les échanges furent plutôt orientés par les sujets-participants euxmêmes. Les retranscriptions furent ensuite codées en suivant les trois étapes de codage proposées par Glaser (1978): «open, selective et theoretical». Ce codage des données - mot par mot facilite l'émergence et la comparaison de concepts à partir des témoignages de chacun des groupes.

\section{Les sujets-participants}

Les sujets-participants A, B, C, D, E et F travaillent tous dans le milieu muséal depuis au moins 4 ans - certains depuis plus de 15 ans. La majorité a travaillé dans plus de 3 institutions culturelles au cours de leur carrière. Tous détiennent un diplôme d'études universitaires, de premier ou de second cycle, soit en histoire de l'art, art visuel, théâtre, littérature et/ou enseignement. Trois sujets-participant(e)s ont une pratique artistique (peinture, performance, écriture) et présentent régulièrement leur travail.

\section{Éléments de définition}

Dans l'ensemble, les témoignages des sujets-participants confirment plusieurs éléments 
de définition déjà bien établis dans la littérature existante ; l'analyse de leurs propos révèle la récurrence de plusieurs grands thèmes, comme le rôle de liaison, de transmission, d'interaction, d'invitation à la participation, à l'expression et à l'appropriation (Caune, 2012 ; Lacerte, 2007 ; Lafortune et Legault, 2012 ; Jacob et Bélanger, 2014). Les sujets-participants mentionnent tous à leur façon l'accompagnement, la notion de «faire le pont», de lier les acquis et les nouvelles connaissances par la «transmission de clés » de lecture et d'observation des œuvres.

Toutefois, les propos recueillis permirent de mettre en lumière le flou constant qui entoure la définition même de la médiation. Les sujets-participants soulignent l'aspect vague de la notion, mais proposent également des pistes de définition, peu souvent verbalisées et pourtant essentielles.

\section{La construction de sens}

Les concepts de construction de sens et de contenu généré prirent une grande importance dans l'analyse des données ; il devint évident que pour ces médiateur(trice)s, leur rôle n'a pas seulement trait à la transmission, mais également à la création de sens et de contenu propre à l'expérience collective des œuvres. A, B et C expliquent que cette construction se déploie en plusieurs temps : lors de la recherche qui précède les visites (travail «en amont»), pendant la rencontre avec les visiteurs et au fil des discussions avec les collègues médiateur(trice)s. L'activité de médiation est un laboratoire de construction de sens où les angles d'approche du médiateur rencontrent ceux des visiteurs pour créer un tout surprenant, unissant à la fois le sens «premier» de l'œuvre, l'expérience qu'en font les visiteurs et leurs interprétations. Ces médiateurs s'adaptent, encadrent l'expérience et effectuent un travail de contextualisation. Les visites se suivent, mais ne se ressemblent pas ! Chaque nouvelle expérience interactive a le potentiel de créer des discours et des situations uniques, inimitables. Dans un cadre idéal, le 
résultat s'éloigne grandement du modèle de transmission didactique du savoir ( «expository») (Hein, 1998, p. 25) pour se diriger plutôt vers un modèle rhizomatique et multivoix. Notons toutefois que ce modèle n'est possible que dans les structures permettant des approches flexibles et personnalisées.

\section{L'oralité, l'éphémérité et la performativité}

L'oralité et l'éphémérité propres au travail de médiation peuvent sembler évidentes, puisque la rencontre ne laisse souvent d'elle-même que des traces intangibles dans les mémoires des visiteurs. Toutefois, comme le soulignent $\mathrm{A}$ et $\mathrm{C}$, ces aspects constituent un noeud, une réalité déterminante. Pour E, il n'est pas étonnant que la médiation connaisse un engouement alors que des pratiques relationnelles, éphémères et performatives s'institutionnalisent ; elles sont de l'ère du temps, issues d'un même contexte où les relations et les tensions relatives à la figure du public sont au coeur des discussions, et fréquemment récupérées par le milieu. La médiation d'œuvres contemporaines présenterait dans les faits plusieurs points communs avec la performance et l'improvisation, en ce sens qu'elle se déploie nécessairement dans l'instant, dans l'immédiateté et très souvent dans l'imprévu ; les sujets-participants soulignent également le grand rôle que joue le rapport personnel (incarné) avec le visiteur et l'usage du corps et de la voix comme outils de communication. Pour ces médiateur(trice)s, la construction de sens et l'oralité vont de pair ; la parole et le langage ramènent à la dimension humaine, à l'expérience et à la mémoire.

\section{L'initiation au lieu}

L'initiation au lieu lui-même est un élément crucial de l'action de médiation et fait donc intrinsèquement partie de sa définition, bien que cet aspect soit rarement verbalisé. En effet, le lieu (le contexte) influence grandement toutes les parties impliquées, c'est-à-dire qu'il détermine 
ce qui sera/pourra être fait, soit par l'entremise de sa structure (mandat, politiques, fonctionnement interne), soit par ses réalités physiques (architecture, espaces). L'environnement qu'est le musée agit comme un intervenant vis-à-vis le visiteur, un peu comme la salle de classe agit auprès de l'élève (third teacher) (Strong-Wilson et Ellis, 2007); il participe à l'apprentissage des visiteurs en déterminant les possibilités et les limites de cet apprentissage. L'analyse des propos recueillis révèle qu'en plus d'initier à l'observation des œuvres, les médiateurs(trice)s invitent leurs groupes à habiter l'espace, à l'étudier, à s'interroger sur le contexte muséal qui encadre leurs expériences des œuvres. Puisque maintes œuvres contemporaines traitent de leur environnement de diffusion et qu'elles sont fréquemment créées en fonction de celui-ci, la médiation du lieu devient un exercice nécessaire à la compréhension de ces œuvres, de leur contexte de création et de diffusion.

\section{Visées et enjeux}

D'aucuns pourraient alors se demander ce que la construction de sens, l'oralité et l'initiation au lieu permettent que des approches différentes ne permettent pas, ou en d'autres mots, ce que sont les objectifs de ces médiateurs, ce qu'ils cherchent à faire advenir. Les visées de la médiation peuvent paraître évidentes, mais en réalité le flou de la définition et l'imprécision des objectifs éducatifs de certaines institutions contribuent à leur complexité. Les témoignages évoquent une différence marquée entre objectifs institutionnels et attentes personnelles des médiateurs, phénomène sur lequel je reviendrai un peu plus loin. Pour l'instant, je me pencherai sur les attentes personnelles des sujets-participants (soulignons qu'elles varient d'une personne à l'autre, mais partagent néanmoins des éléments communs).

\section{Dimension critique}

Dans certains contextes, la médiation peut être perçue comme une finalité, se suffisant à 
elle-même, au point de se retrouver dépourvue de dimension critique ou de visées citoyennes (Lafortune, 2012). La médiation prône l'interaction, certes, mais à quoi sert cette interactivité, à quoi participe-t-elle? Pour ces sujets-participants, l'interactivité est à la fois l'essence même de la pratique et un moyen d'accéder à des visées plus vastes. Ils partagent une volonté de situer leurs actions dans une réflexion critique sur les œuvres, le rapport aux visiteurs et le lieu. Bien que les termes «démocratie culturelle»n'aient pas été fréquemment employés, ils sont toutefois suggérés par l'utilisation des termes «autonomie », «prise de pouvoir », « découvrir par soimême », «s'approprier l'espace et les œuvres », etc. L'analyse des six témoignages illustre une volonté très marquée chez les sujets-participants de faire naître chez le visiteur-citoyen une volonté d'aller au-delà des premières impressions, de faire sens collectivement, de vivre une expérience sensible, de prendre position, de commenter et de débattre. Le ludisme et le plaisir de la rencontre, bien que présents, ne représentent pas la seule finalité.

\section{Rapport aux visiteurs, aux œuvres et au savoir}

Les médiateurs rencontrés se sont montrés très sensibles à la manière dont leur rôle teinte l'expérience du visiteur, à la façon dont leur présence transforme le contexte de présentation des œuvres. Cette sensibilité leur permet de réaliser la dimension duelle de leur rôle, entre figure de détenteur du savoir et figure d'ouverture. L'échange tel qu'ils le rêvent peut-il vraiment avoir lieu dans un contexte muséal? Pour ce faire, la hiérarchie des rôles éducateur/apprenant doit-elle s'amenuiser ou même disparaître? Et inversement, est-il souhaitable d'occulter complètement toute prévalence du discours du médiateur sur celui des visiteurs? Là se trouve un autre noeud important de la médiation de l'art contemporain, dans son rapport aux modes traditionnels de transmission du savoir, et à la notion même de savoir.

Il semble qu'en ce sens, les médiateur(trice)s de l'art contemporain/actuel agissent sur un 
terrain plus mouvant que leurs collègues des musées de sciences ou d'histoire. D'une part, la nature des œuvres elles-mêmes encourage une négociation entre plusieurs sens possibles, entre théorie et expérience(s). L'œuvre contemporaine étant généralement ouverte (Eco, 1989), elle partage des informations - plutôt que des messages univoques - qui mènent à une abondance de lectures possibles. Dans un contexte idéal, la médiation s'adapte à cette réalité en accueillant ces lectures et les éléments générés par les visiteurs. Toutefois, les limites de cette ouverture sont fréquemment remises en question dans les écrits théoriques, notamment quant à la notion de vulgarisation. L'ouverture - aux interprétations, à la parole du visiteur - et le devoir d'accessibilité pourraient mener à la simplification à outrance d'œuvres complexes, à la «banalisation conceptuelle » (Janelle, 1994, p.10) ou encore la déclassification de pratiques jugées difficiles (Lafortune, 2012). Toutefois, l'abandon du modèle «construction de sens » semblerait trahir les objectifs de la médiation et la pluralité des œuvres présentées.

Les codes «auto-analyse » et «transparence » firent rapidement leur apparition lors du codage des données et furent regroupés dans une catégorie étiquetée Introspection. Les sujetsparticipants se sont montrés conscients de la dualité «contenu / expérience » et semblent tous miser sur une stratégie prônant l'équilibre (entre fidélité à la théorie/histoire de l'art et ouverture aux interprétations) et l'auto-analyse constante. L'outil de prédilection est un sens critique aiguisé vis-à-vis l'acte de médiation et une vaste connaissance de ses enjeux : savoir se remettre en question, se «voir agir », s'interroger sur les impacts de ses interventions, sur ce qui est généré à long terme, ajuster ses approches, reconnaître qui - de l'œuvre ou du visiteur - est mis de l'avant, et finalement établir un équilibre entre contenu et expérience collective de l'œuvre. Les participants reconnaissent à la fois l'importance et la difficulté de cette entreprise, soulignant qu'aucune manœuvre ne garantit absolument l'atteinte de ces objectifs. 


\section{Déconstruire les rôles}

Cette auto-analyse vise également la prise de conscience de la place qu'occupe le médiateur dans l'échange. Certains sujets-participants abordent ces problématiques en les verbalisant clairement et en faisant preuve d'une grande transparence. Par exemple, D encourage ouvertement les visiteurs à questionner les dynamiques éducateur/apprenant et à inverser les rôles, tentant ainsi de déconstruire les dynamiques hiérarchiques au sein du groupe: « Je n'ai pas le savoir »(Farley, 2015, p. 65). « Pourquoi n'échangerions-nous pas nos places?» (Farley, 2015, p. 44). B, C et F rappellent qu'ils ne cherchent pas à convaincre ou imposer, mais à offrir des outils, un espace à prendre, une rencontre à échelle humaine et une arène d'échange et de débats. Même les frictions, les confrontations et l'absence de consensus ont leur place dans le contexte muséal!

\section{Identification de défis et de tensions}

La question de la médiation est complexe puisqu'elle implique à la fois des visées institutionnelles (lieux de diffusion) et des attentes personnelles (médiateurs). Or, les discussions avec les sujets-participants suggèrent que les attentes de l'un et l'autre, sans être toujours parfaitement irréconciliables, se rencontrent néanmoins difficilement. Cinq des six sujetsparticipants affirment que leur situation au sein des institutions représente un obstacle important : objectifs institutionnels imprécis, rôle peu compris et peu (re)connu, importance parfois mitigée aux yeux de leurs supérieurs, sentiment de dénigrement, précarité de l'emploi, instabilité des revenus, etc. Cependant, les activités de médiation se font de plus en plus présentes et un discours officiel presque triomphaliste les entoure. Pourquoi cette double réalité ? Entre la présence de la médiation - sa nécessité claironnée par maints intervenants culturels - et sa dévalorisation, que se passe-t-il? 


\section{Valorisation et (re)connaissance}

D’une part, il est possible que la médiation souffre de sa propre oralité; bien que celle-ci soit intrinsèque au travail, il est possible que l'absence de traces «tangibles » - de l'écrit ou de l'objet - rende difficiles sa justification et sa valorisation. Certains sujets-participants supputent que cette intangibilité et cette éphémérité participeraient au manque de reconnaissance de leur travail - possiblement au flou qui l'entoure. L'essentiel de leurs actions demeurant méconnues, les aptitudes que requiert leur profession tendent à être minimisées. Pour les musées et galeries, il peut donc s'avérer tentant d'opter pour une main-d'œuvre bénévole, surtout si des impératifs budgétaires encouragent ce choix. Les sujets-participants déplorent toutefois le fait que ce choix découle parfois d'une méconnaissance de leur travail et de ses impacts, voire d'un certain dénigrement, plutôt que de réelles obligations d'ordre pécuniaire. Il en résulte un statut précaire pour ces médiateurs(trice)s, en plus d'un sentiment d'insatisfaction (modéré à important) de leurs visées personnelles.

\section{Cloisonnement et hiérarchie}

Les codes hiérarchie, cloisonnement et idéologie institutionnelle sont récurrents dans l'analyse des données. Bien que le fonctionnement interne de chaque institution soit unique, la nébulosité des objectifs éducatifs de certains établissements et une forte hiérarchie entre les paliers mèneraient à: 1) des disparités entre les objectifs des médiateur(trice)s et celles des lieux d'embauche; 2) des décisions allant à l'encontre des visées de la médiation. De manière concrète, ces divergences affectent directement la façon dont les médiateur(trice)s pourront aborder les problématiques mentionnées plus haut, comme la question de la vulgarisation, le rapport aux visiteurs, l'ouverture et la flexibilité dont ils pourront faire preuve - en somme, leur philosophie éducative. Ces tensions mentionnées par les sujets-participants rappellent cet énoncé de Jean- 
Marie Lafortune (2012): «Malgré leur précaution, les médiateurs culturels contribuent souvent à l'avènement d'une situation contraire à celle qu'ils cherchent précisément à faire advenir » (p. 76).

Il convient toutefois de noter que toutes les expériences ne sont pas analogues; il semblerait que les plus petits organismes tendent à valoriser davantage le travail des médiateur(trice)s et à accorder une plus grande importance à leur expertise et à leur voix. Il se peut que la structure même de ces organismes en soit la cause, puisqu'il s'agit de petites équipes, moins hiérarchisées, où les médiateur(trice)s occupent également des postes de gestion, de création de programmes et de coordination. Dans ces contextes, les pratiques artistiques des médiateurs/artistes sont également davantage valorisées et mises à contribution.

\section{Conclusion}

Les discussions avec les six sujets-participants ont permis d'étudier certains aspects de leur travail et de proposer des pistes de définition basées sur l'expérience. De plus, ces échanges ont mis en lumière le statut profondément paradoxal de la médiation de l'art contemporain en milieu muséal, entre visées personnelles et impératifs institutionnels, entre dimension critique et divertissement, entre omniprésence et précarité. Les sujets-participants décrivent un «entredeux » à la fois riche et inconfortable; riche de leurs échanges uniques avec les visiteurs, et inconfortable dû à la méconnaissance de leur rôle, à leur relative «invisibilité » dans certaines institutions et aux divergences idéologiques rencontrées.

En somme, il n'est pas suffisant de se questionner sur les pratiques de terrain des médiateur(trice)s, mais il est également essentiel de se questionner sur les contextes spécifiques des lieux qui chapeautent ces initiatives culturelles. Plusieurs questions méritent d'être soulevées: les objectifs institutionnels et les objectifs citoyens/artistiques/critiques sont-ils 
réconciliables? La médiation s'inscrit-elle réellement dans le projet institutionnel, et comment? Les activités que l'on présente comme de la « médiation » en sont-elles vraiment? Est-il pensable de mettre de côté la notion de résultats quantifiables? Les médiateur(trice)s ont-ils réellement la possibilité de s'exprimer et de prendre position en tant qu'acteurs culturels? Ont-ils les tribunes pour le faire? Les témoignages des sujets-participants indiquent qu'une meilleure compréhension du travail de médiation en contexte muséal est nécessaire à la valorisation de leur pratique et à son développement. Si le monde académique se penche depuis longtemps sur la «teneur idéologique » (Caune, 2012) des discours et des dispositifs de la médiation, il est crucial que les lieux de diffusion participent au même exercice.

\section{Références}

Caune, J. (2012). Préface. La médiation culturelle: Le sens des mots et l'essence des pratiques. (p. VII- XX). Montréal: Presses de l’Université du Québec.

Eco, U. (1989). The Open Work. (A. Cancogni, trad.) U.K.: Hutchinson Radius.

Farley, M. (2015). Réconcilier l'irréconciliable: l'expérience de terrain des médiatrices et médiateurs de l'art contemporain (Mémoire de maîtrise) http://spectrum.library.concordia.ca/979862/1/Farley_MA_S2015.pdf

Glaser, G. B. (1978). Theoretical Sensitivity: Advances in the Methodology of Grounded Theory. California: The Sociology Press.

Hein, G. E. (1998). Learning in the Museum. New York: Routledge.

Jacob \& Bélanger (2014). Les effets de la médiation culturelle: Participation, expression, changement. Document de synthèse, étude partenariale réalisée à Montréal de 2012 à 2013.

Janelle, S. (1994). L'art de vulgariser. ETC, no 27, (p. 10-12). Consulté à l'adresse 
http://id.erudit.org/iderudit/35665ac

Lacerte, S. (2007). La médiation de l'art contemporain: Pour qui? Pourquoi? Consulté à l'adresse http://montreal.mediationculturelle.org/wpcontent/uploads/2010/04/mediation_pourqui_p ourquoi_lacerte.pdf

Lafortune, J.M. (2012). Enjeux et limites de la médiation culturelle. La Médiation culturelle: Le sens des mots et l'Essence des Pratiques. (p. 61-78) Montréal: Presses de l'Université du Québec.

Lafortune, J. M. \& Legault, C. (2012). Acteurs et Dispositifs de la médiation culturelle. La Médiation culturelle: Le sens des mots et l'Essence des Pratiques. (p. 39-59). Montréal: Presses de l’Université du Québec.

Stern, P. N. \& Porr, C. J. (2011). Essentials of Accessible Grounded Theory. California: Left Coast Press.

Strong-Wilson, T. \& Ellis, J. (2007). Children and Place: Reggio Emilia’s Environment as Third Teacher. Theory into Practice. 46:1, 40-47. doi: 10.1080/00405840709336547, extrait de http://dx.doi.org/10.1080/00405840709336547

Urquhart, C. (2013). Grounded Theory for Qualitative Research: A Practical Guide. Los Angeles, CA: Sage Publications. 